\title{
FLOT2 overexpression is associated with the progression and prognosis of human colorectal cancer
}

\author{
TAIYUAN LI* , CHUANLIN CAO*, QIANGQIANG XIONG and DONGNING LIU \\ Department of General Surgery, The First Affiliated Hospital of Nanchang University, Nanchang, Jiangxi 330006, P.R. China
}

Received September 11, 2016; Accepted November 1, 2018

DOI: $10.3892 / \mathrm{ol} .2019 .9882$

\begin{abstract}
Altered expression of flotillin-2 (FLOT2) has been identified in certain types of cancer, including breast cancer and melanoma; however, to the best of our knowledge, the association between the FLOT2 expression level and colorectal cancer (CRC) remains to be determined. The aim of the present study was to investigate the clinical and prognostic significance of FLOT2 in CRC. The expression of FLOT2 was determined in seven CRC cell lines and one normal colon cell line, and it was identified that FLOT2 was increased in CRC cell lines, suggesting that FLOT2 exhibited an association with CRCs. In addition, FLOT2 protein levels were investigated in primary CRC tissues and corresponding non-cancerous colon tissues from 8 patients. Compared with non-cancerous tissues, FLOT2 protein was apparently upregulated in CRC tissues. To validate this result, an immunohistochemistry assay was performed and it was identified that FLOT2 levels were increased in CRC tissues. Clinical analysis identified that increased expression of FLOT2 was associated with the depth of invasion, lymph node metastasis, distant metastasis and American Joint Committee on Cancer stage of CRCs. Furthermore, multivariate analysis using the Cox regression model indicated that increased FLOT2 expression was an independent prognostic factor in patients with $\mathrm{CRC}(\mathrm{P}=0.013)$. Taken together, the results of the present study suggest that overexpression of FLOT2 contributes to the progression of $\mathrm{CRC}$ and indicate that FLOT2 is a novel target for the treatment of CRC.
\end{abstract}

\section{Introduction}

According to statistical data from 2012, colorectal cancer (CRC) is the third most popular cancer and the fourth most

Correspondence to: Dr Dongning Liu, Department of General Surgery, The First Affiliated Hospital of Nanchang University, 17 Yongwaizheng Street, Nanchang, Jiangxi 330006, P.R. China

E-mail: liudongning1982@sina.com.cn

${ }^{*}$ Contributed equally

Key words: flotillin-2, colorectal cancer, prognosis, biomarker common cause of cancer mortality globally (1). It is estimated that $>1$ million novel cases of colorectal carcinoma are diagnosed annually $(2,3)$. Despite the improvement in surgical, radiotherapeutic and chemotherapeutic regimens, $\sim 50 \%$ of patients with CRC relapse within 5 years of treatment. Evidence suggests that this is due to distant metastasis (4). However, the responsiveness of patients to treatment is diverse because of the accumulation of multiple genetic mutations involving critical genes that govern cell proliferation (5). Therefore, a novel biomarker for CRC is urgently required to help diagnose the disease at an early stage and determine the treatment response in patients with CRC, thereby enabling an accurate prediction of the prognosis of patients.

Lipid rafts, specialized domains in cell membranes, are involved in a variety of cell signal transductions (6). The flotillin family of proteins, including two isoforms flotillin-1 (FLOT1) and flotillin-2 (FLOT2), are important markers of lipid rafts $(7,8)$. It has been reported that flotillin proteins ubiquitously express and serve important functions in a number of biological processes, including cell adhesion, endocytosis, actin reorganization, signaling transduction, phagocytosis and actin cytoskeleton dynamics (9-13). Furthermore, FLOT2 has been demonstrated to be upregulated and involved in progression and development in several types of cancer, including breast cancer and melanoma (14-17). A previous microarray result has identified that FLOT2 was upregulated in a cluster of breast tumors (14). FLOT2 protein exhibited low expression in non-tumorigenic cell lines, whereas high expression was identified in certain metastatic melanoma cell lines in vitro (15). Consistently, the ectopic expression of FLOT2 was associated with the progression of human melanoma in vivo (15). A previous study revealed that knockdown of FLOT2 attenuated the proliferation and metastasis of a human breast cancer cell line (16). It also identified that the increased FLOT2 protein expression was associated with poor outcomes in patients with breast cancer and that it could be used as a biomarker for breast cancer progression (17). Although the increase in FLOT2 is associated with a number of types of tumor, to the best of our knowledge, its function in regulating CRCs remains to be determined.

To determine the association between FLOT2 expression and CRCs, cell lines and patient tissues were analyzed. It was revealed that FLOT2 was apparently increased in CRC cell lines and CRC tissues. The upregulated FLOT2 was associated with invasion, lymph node metastasis, distant metastasis 
and patient survival. Taken together, the results of the present study suggest that FLOT2 acts as a biomarker for CRC and that FLOT2 may be a potential drug target for the clinical treatment of CRC.

\section{Materials and methods}

Patient information and specimen collection. A total of $8 \mathrm{CRC}$ and corresponding normal mucosa tissue samples ( $>10 \mathrm{~cm}$ away from the edge of the CRC) were obtained from patients with CRC (age range, 56-72 years; sex, 5 male and 3 female) within 30 min of resection at the First Affiliated Hospital of Nanchang University (Nanchang, China) between April 2017 and June 2017, and then snap-frozen in liquid nitrogen and stored at $-80^{\circ} \mathrm{C}$ until use. Formaldehyde-fixed and paraffin-embedded CRC tissue blocks $(n=180)$ were obtained from the stored files of the Department of General Surgery, The First Affiliated Hospital of Nanchang University (Nanchang, China) collected between January 2006 and December 2008. All patients had undergone pre-operative clinical staging assessment with magnetic resonance imaging. No patients had received chemotherapy or radiotherapy prior to surgery. The various clinicopathological parameters [age, sex, tumor size, tumor location, tumor differentiation, histological types, depth of invasion, lymph node metastasis and distant metastasis and American Joint Committee on Cancer (AJCC) stage] were obtained from histopathology records. The stage of colorectal cancer was described according to the 7th edition of the AJCC Tumor-Node-Metastasis classification of malignant tumors (18). Use of CRC specimens and matched normal specimens for the present study was approved by the Ethics Committee of the First Affiliated Hospital of Nanchang University.

The 180 patients included 93 men and 87 women aged between 21 and 92 years (mean, 61.5 years). The patients were followed-up until mortality or to the end of the follow-up period (30 November 2015). The follow-up durations, ranging between 2 and 92 months, were available for all patients and the median patient survival time was 65 months. Distant metastasis occurred in 24 cases $(13.4 \%)$, including 5 cases to the peritoneum, 17 cases to the liver and 2 cases to the bone.

Cell culture. A total of seven CRC cell lines (HT-29, SW480, Lovo, SW1116, SW620, Colo205 and DLD1) and one normal colon cell line (FHC) were purchased from the American Type Culture Collection (Manassas, VA, USA). Cells were cultured in RPMI-1640 medium supplemented with $10 \%$ fetal bovine serum, $1 \%$ glutamine and $1 \%$ penicillin/streptomycin (all from Thermo Fisher Scientific, Inc., Waltham, MA, USA) at $37^{\circ} \mathrm{C}$ in a humidified incubator containing an atmosphere of $5 \% \mathrm{CO}_{2}$.

RNA isolation, reverse transcription and reversetranscription-quantitative polymerase chain reaction $(R T-q P C R)$. Cultured cells or tissues were lysed with TRIzol ${ }^{\circledR}$ reagent (Invitrogen; Thermo Fisher Scientific, Inc.) for RNA extraction, according to the manufacturer's protocol. The extracted RNA was pretreated with RNase-free DNase, and $1 \mu \mathrm{g}$ RNA from each sample was reverse transcribed with TaqMan reverse transcription reagents and random hexamer primers (Applied Biosystems; Thermo Fisher Scientific, Inc.).
The RNA sample was incubated in a thermocycler for $1 \mathrm{~h}$ at $40^{\circ} \mathrm{C}$ and then denatured at $95^{\circ} \mathrm{C}$ for 2 min prior to incubation on ice. For PCR-mediated amplification of FLOT2 cDNA using FLOT2-specific primers, a denaturation step at $95^{\circ} \mathrm{C}$ for 10 min was followed by 30 cycles consisting of denaturation at $95^{\circ} \mathrm{C}$ for $60 \mathrm{sec}$, primer annealing at $55^{\circ} \mathrm{C}$ for $30 \mathrm{sec}$, and primer extension at $72^{\circ} \mathrm{C}$ for $30 \mathrm{sec}$. On completion of the cycling, a final extension step at $72^{\circ} \mathrm{C}$ for $5 \mathrm{~min}$ was performed before the reaction was stopped and stored at $4^{\circ} \mathrm{C}$. qPCR was performed on an ABI Fast 7500 instrument using Maxima SYBR Green qPCR Master mix (Thermo Fisher Scientific, Inc.). The $2^{-\Delta \Delta C q}$ method was used for relative quantification (19). The primers used in this study were designed using Primer Express version 3.0 (Applied Biosystems; Thermo Fisher Scientific, Inc.). The primer pairs used were as follows: FLOT2, 5'-CCC CAGATTGCTGCCAAA-3' (forward) and 5'-TCCACTGAG GACCACAATCTCA-3' (reverse); and GAPDH, 5'-ACCACA GTCCATGCCATCAC-3' (forward) and 5'-TCCACCACC CTGTTGCTGTA-3' (reverse).

Western blot analysis. Cells at 70-80\% confluence were washed twice with ice-cold PBS and lysed on ice in radioimmunoprecipitation assay buffer (RIPA; Cell Signaling Technology, Inc., Danvers, MA, USA) containing complete protease inhibitor cocktail (Roche Applied Science, Mannheim, Germany). Fresh tissue samples were ground to powder in liquid nitrogen and lysed with SDS-PAGE sample buffer. Protein concentration was measured using a BCA protein assay kit (Pierce; Thermo Fisher Scientific, Inc.). Equal protein samples $(20 \mu \mathrm{g})$ were separated by SDS-PAGE (10.5\% gels) and transferred to polyvinylidene fluoride membranes (Merck KGaA, Darmstadt, Germany). Membranes were blocked with 5\% fat-free milk in Tris-buffered saline containing $0.1 \%$ Tween-20 for $1 \mathrm{~h}$ at room temperature. Membranes were incubated with rabbit anti-FLOT2 (1:1,000; cat. no. ab96507; Abcam, Cambridge, UK) overnight at $4^{\circ} \mathrm{C}$, and then with horseradish peroxidase-conjugated rabbit anti-mouse secondary antibodies (1:2,000, cat. no. ab6728; Abcam). FLOT2 expression was detected using ECL prime western blotting detection reagent (GE Healthcare, Chicago, IL, USA) according to the manufacturer's instructions. GAPDH (mouse anti-GAPDH; 1:1,000; cat. no. ab9484; Abcam) was used as a loading control.

Immunohistochemical (IHC) staining. Samples were fixed in $4 \%$ formaldehyde solution, embedded in paraffin blocks, cut into 4- $\mu$ m-thick sections and mounted on glass slides. Each slide was dewaxed in xylene and rehydrated in a graded alcohol series, followed by boiling in $10 \mathrm{mmol} / \mathrm{l}$ citrate buffer ( $\mathrm{pH}$ 6.0) for antigen retrieval. Following inhibition of endogenous peroxidase activities for $30 \mathrm{~min}$ with methanol containing $0.3 \% \mathrm{H}_{2} \mathrm{O}_{2}$, the sections were blocked with $2 \%$ bovine serum albumin for $30 \mathrm{~min}$ and incubated overnight at $4^{\circ} \mathrm{C}$ with rabbit anti-FLOT2 antibody (1:100). Following washing three times with PBS, the slides were incubated with horseradish peroxidase-conjugated rabbit anti-mouse antibody (1:400; cat. no. ab6728; Abcam) for $30 \mathrm{~min}$, followed by reaction with diaminobenzidine and counterstaining with Mayer hematoxylin. The negative control consisted of non-specific mouse immunoglobulin $G$ rather than the primary antibody. 
The results of immunostaining were evaluated by two observers without prior knowledge of the clinical information of the patients, on the basis of the proportion of positively stained tumor cells and the intensity of staining (17). The scores attributed by the two independent investigators were averaged. The proportion of tumor cells was scored as follows: 1 ( $<10 \%$ positive tumor cells), 2 (10-50\% positive tumor cells), $3(50-75 \%$ positive tumor cells) and 4 ( $>75 \%$ positive tumor cells). The intensity of staining was graded according to the following criteria: 0 (no staining), 1 (weak staining; light yellow), 2 (moderate staining; yellow brown) and 3 (strong staining; brown). The staining index was calculated as the product of the proportion of positive cells and the staining intensity score. Threshold values for FLOT2 were chosen on the basis of a measure of heterogeneity using the log-rank test with respect to overall survival (OS). An optimal threshold value was identified as follows: A staining index score of $\geq 6$ was used to define tumors with high FLOT2 expression and $\leq 4$ indicated low FLOT2 expression.

Statistical analysis. All statistical analyses were performed using the SPSS statistical software package (version 19.0; IBM Corp., Armonk, NY, USA). Differences in FLOT2 expression between normal colon cell and colorectal cancer cell lines were analyzed using an independent t-test. Differences in FLOT2 expression between colorectal cancer tissues and adjacent non-cancerous tissues in the same patient were analyzed using a paired t-test. The associations between FLOT2 expression and clinicopathological characteristics were analyzed using the Pearson $\chi^{2}$ test. Survival rates were calculated according to the Kaplan-Meier method, and differences were evaluated using the log-rank test. The Cox proportional hazards regression model was used to determine the hazard ratio and identify factors that independently predict survival. $\mathrm{P}<0.05$ in all cases was considered statistically to indicate a statistically significant difference.

\section{Results}

FLOT2 is highly expressed in seven CRC cell lines. To investigate the function of FLOT2 in CRCs, the expression of FLOT2 in CRC cell lines was determined. The expression levels of FLOT2 mRNA and protein were compared in seven CRC cell lines (HT-29, SW480, Lovo, SW1116, SW620, Colo205 and DLD1) and one normal colon cell line (FHC). FLOT2 protein was highly expressed in CRC cell lines and only weakly expressed in FHC (Fig. 1A). In addition, FLOT2 mRNA expression was at least 4-fold higher in CRC cell lines compared with FHC cells (Fig. 1B). Taken together, these results indicate that CRC cell lines exhibit high FLOT2 expression, indicating that FLOT2 possibly serves a function in CRCs.

Expression of FLOT2 is increased in CRC tissues. Since FLOT2 is increased in CRC cell lines, it was investigated whether this was the case in vivo. CRC tissue samples and adjacent non-cancerous tissues were obtained from 8 patients with CRC. FLOT2 protein was upregulated in CRC samples compared with matched controls (Fig. 2A). Consistent with this data, FLOT2 mRNA was also expressed at higher levels in
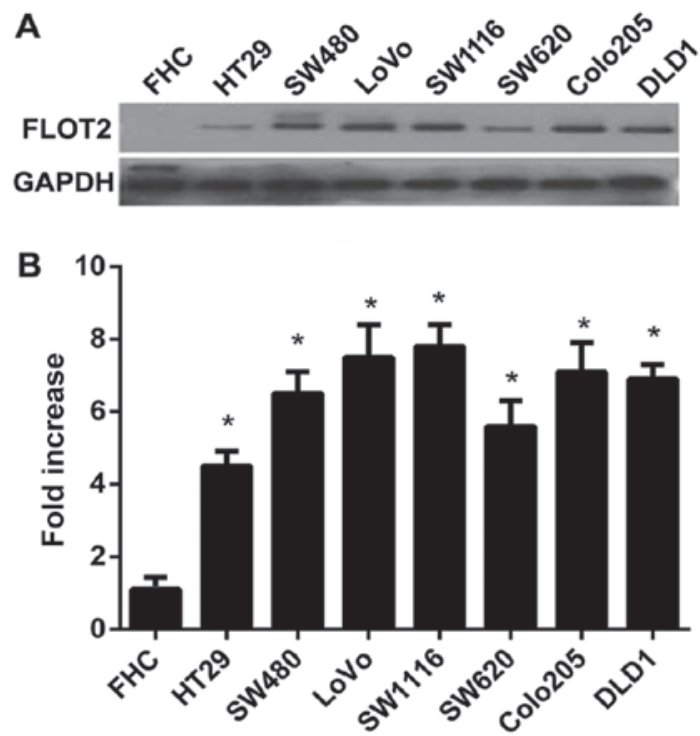

Figure 1. Expression of FLOT2 is upregulated in colorectal cancer cell lines. (A) The normal colon cell (FHC) and colorectal cancer cell lines (HT-29, SW480, Lovo, SW1116, SW620, Colo205 and DLD1) were analyzed by western blotting. GAPDH served as a loading control. (B) FLOT2 mRNA expression in the various cell lines was quantified using the reverse transcription-quantitative polymerase chain reaction. Expression level were normalized to GAPDH and presented as the mean \pm standard error of the mean. ${ }^{*} \mathrm{P}<0.05$ vs. FHC. FLOT2, flotillin-2.

A

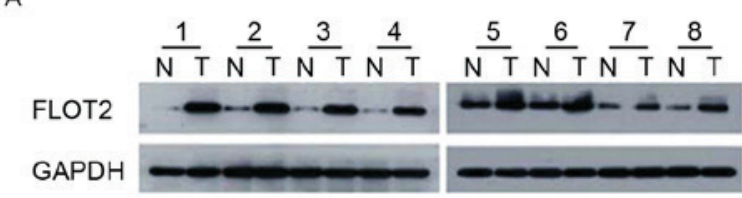

B

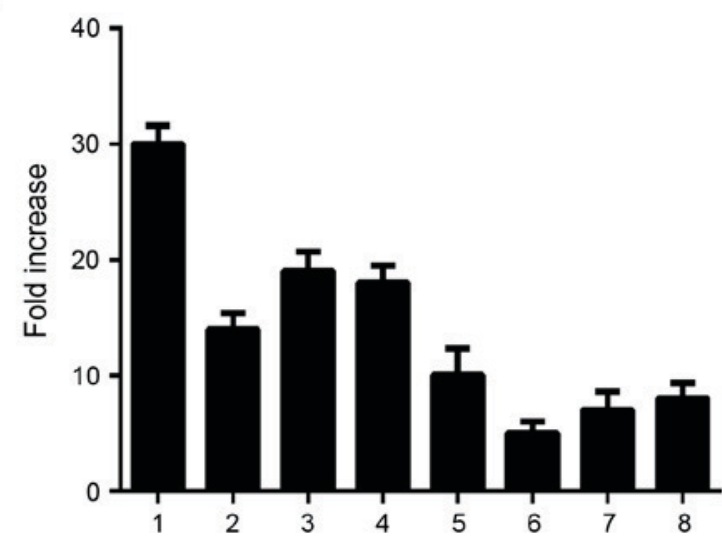

Figure 2. Expression of FLOT2 is increased in colorectal cancer tissues. (A) Tissues were analyzed by western blotting. GAPDH served as a loading control. (B) Mean T/N ratios of FLOT2 mRNA expression were determined following quantification using the reverse transcription-quantitative polymerase chain reaction. Results are expressed as the mean \pm standard error of the mean. $\mathrm{T}$, tumor tissues; $\mathrm{N}$, adjacent non-tumor tissues.

all CRC tissue samples compared with adjacent non-cancerous tissues, with a differential expression that ranged from 6.3-fold to 30.2 -fold (Fig. 2B). These data indicated that FLOT2 is upregulated in vitro and in vivo.

In addition, IHC staining was used to examine the expression of FLOT2 in 180 paired paraffin-embedded CRC tissues. 


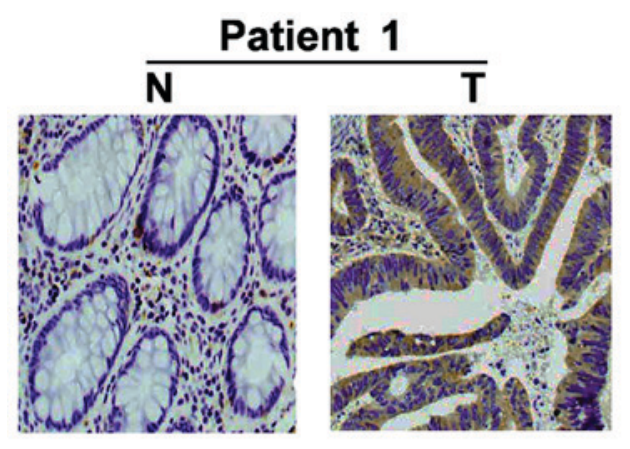

Patient 3

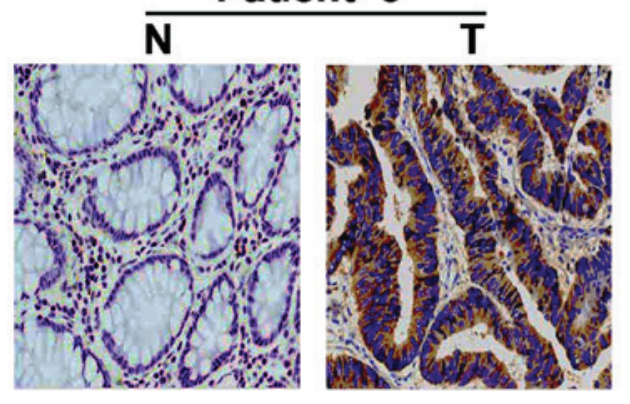

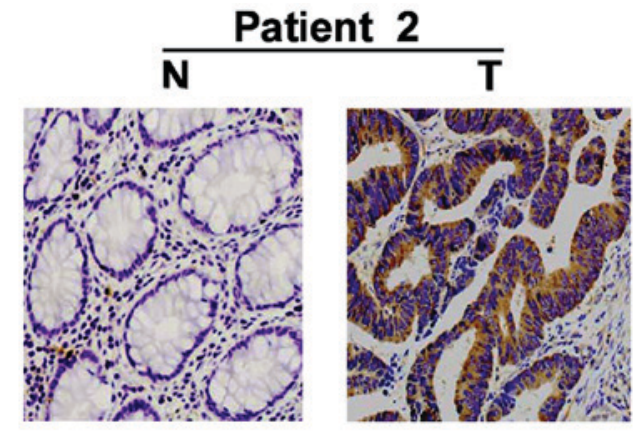

Patient 4

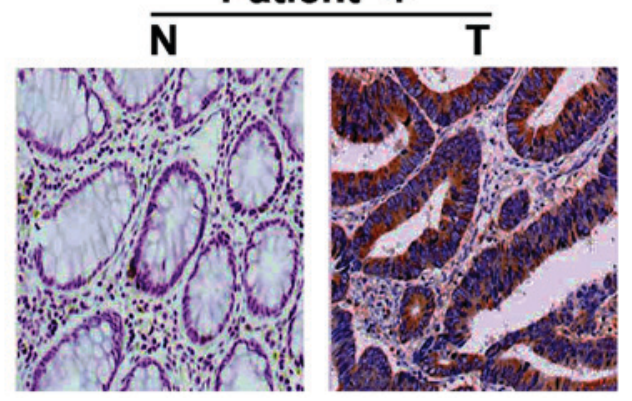

Figure 3. Colorectal cancer tissues exhibit increased FLOT2 expression, as revealed by immunohistochemistry. FLOT2 stained brown, with weaker expression staining yellow. FLOT2, flotillin-2; T, tumor tissues; N, adjacent non-tumor tissues.

FLOT2 protein was predominantly localized on the plasma membrane (Fig. 3). In the 180 samples, a high level of FLOT2 protein was detected in 119 samples (66.11\%; Table I) and low or no staining was observed in 61 tumor samples $(33.89 \%$; Table I). As presented in Fig. 3, no or weak signals were detected in the adjacent non-cancerous tissues and normal colorectal tissues. In contrast, FLOT2 was highly expressed in CRC tissues. Taken together, these observations indicate that FLOT2 is overexpressed in CRC tissues.

Association of FLOT2 expression and clinicopathological features of patients with CRC. FLOT2 expression was identified to be increased in CRC tissues, suggesting that certain connections exist between FLOT2 levels and CRC. To investigate this possibility, statistical analysis was performed. Associations between FLOT2 expression and the clinicopathological characteristics of CRC were identified, including depth of invasion $(\mathrm{P}=0.024)$, lymph node metastasis $(\mathrm{P}=0.005)$, distant metastasis $(\mathrm{P}=0.008)$ and AJCC stage $(\mathrm{P}=0.011)$ (Table $\mathrm{I})$. In contrast, no apparent associations were identified between FLOT2 expression and other factors, including sex, age, tumor size, tumor location, histological types and tumor differentiation (Table I). These results indicate that FLOT2 may serve as a biomarker for the diagnosis of CRC.

Association between FLOT2 expression and prognosis in patients with CRC. Next, whether the expression of FLOT2 regulates the survival of patients was investigated. The Kaplan-Meier analysis and the log-rank test were used to analyze the effect of FLOT2 expression on survival time. Survival curves revealed that the patients with CRC with high FLOT2 expression had worse OS and disease-free survival (DFS) compared with those with low FLOT2 expression
$(\mathrm{P}=0.001$ and $\mathrm{P}<0.001)$ (Fig. 4A and $\mathrm{B})$. The survival rate of patients with CRC with high FLOT2 expression decreased in the pT3-4 subgroup, but not in the pT1-2 subgroup $(\mathrm{P}<0.001$ and $\mathrm{P}=0.519$ ) (Fig. 4C and D). Similar results were identified in the AJCC stage I-II and III-IV subgroups $(\mathrm{P}<0.001$ and $\mathrm{P}=0.679$ ) (Fig. $4 \mathrm{E}$ and $\mathrm{F}$ ). As presented in Table II, the univariate survival analysis indicated that the OS was markedly associated with FLOT2 expression, depth of invasion, lymph node metastasis, distant metastasis and AJCC stage. Furthermore, multivariate survival analysis was performed using Cox proportional hazard model to confirm that FLOT2 expression level, lymph node metastasis, distant metastasis and AJCC stage were independent poor prognostic factors for OS of CRC.

\section{Discussion}

Nearly $50 \%$ of patients with CRC succumb owing to distant metastasis, particularly to the liver. The patients with liver metastasis are not suitable for surgical treatment, thus the survival rate of patients with liver metastasis is $<10 \%$ (20). Conversely, the prognosis of patients with $\mathrm{CRC}$ remains poor. Therefore, there is an urgent requirement to identify a novel biomarker for CRC.

In the present study, it was identified that the expression of FLOT2 was apparently upregulated in CRC cell lines compared with in a normal colon cell line. Furthermore, using an in vivo assay, it was revealed that the CRC tissues exhibited high FLOT2 expression, whereas adjacent normal tissues exhibited low FLOT2 expression. These results clearly demonstrated that FLOT2 was markedly overexpressed in human CRC tissues compared with in normal colorectal epithelium. Although the underlying molecular mechanisms leading to FLOT2 overexpression in human CRC tissues 
Table I. Association between FLOT2 expression and other clinicopathological features in colorectal carcinoma.

\begin{tabular}{|c|c|c|c|}
\hline \multirow[b]{2}{*}{ Characteristic } & \multicolumn{2}{|c|}{ FLOT2 expression } & \multirow[b]{2}{*}{ P-value } \\
\hline & $\begin{array}{c}\text { Low/no } \\
(\mathrm{n}=61)\end{array}$ & $\begin{array}{c}\text { High } \\
(\mathrm{n}=119)\end{array}$ & \\
\hline Sex & & & 0.979 \\
\hline Male & 32 & 61 & \\
\hline Female & 29 & 58 & \\
\hline Age, years & & & 0.829 \\
\hline$<60$ & 17 & 35 & \\
\hline$\geq 60$ & 44 & 84 & \\
\hline Tumor size, $\mathrm{cm}$ & & & 0.730 \\
\hline$<5$ & 25 & 50 & \\
\hline$\geq 5$ & 36 & 69 & \\
\hline Tumor location & & & 0.497 \\
\hline Colon & 20 & 30 & \\
\hline Rectum & 41 & 89 & \\
\hline Histological type & & & 0.189 \\
\hline NMC & 58 & 94 & \\
\hline $\mathrm{MC}$ & 3 & 25 & \\
\hline Differentiation & & & 0.517 \\
\hline Well & 16 & 25 & \\
\hline Moderate & 22 & 35 & \\
\hline Poor & 23 & 59 & \\
\hline Depth of invasion & & & 0.024 \\
\hline $\mathrm{T} 1$ & 18 & 19 & \\
\hline $\mathrm{T} 2$ & 19 & 26 & \\
\hline $\mathrm{T} 3$ & 14 & 34 & \\
\hline $\mathrm{T} 4$ & 10 & 40 & \\
\hline Lymph node metastasis & & & 0.005 \\
\hline No & 33 & 41 & \\
\hline $\mathrm{N} 1-2$ & 28 & 78 & \\
\hline Distant metastasis & & & 0.008 \\
\hline M0 & 55 & 101 & \\
\hline M1 & 6 & 18 & \\
\hline AJCC stage & & & 0.011 \\
\hline $\mathrm{I}+\mathrm{II}$ & 30 & 46 & \\
\hline III+IV & 31 & 73 & \\
\hline
\end{tabular}

Distant metastasis includes peritoneum, liver and bone metastasis. FLOT2, flotillin 2; AJCC, American Joint Committee on Cancer; MC, mucinous adenocarcinoma; NMC, non-mucinous adenocarcinoma.

are unclear, FLOT2 is proposed as a potential biomarker for the diagnosis of CRC. Given that the mRNA levels of FLOT2 were also increased in CRC tissues, we hypothesize that gene amplification may account for FLOT2 overexpression. This possibility requires investigation in future studies using techniques including next-generation sequencing and genome-wide association studies.

The association between the expression of FLOT2 and the clinical characteristics of patients with CRC was investigated.
An association between FLOT2 expression and the depth of invasion, lymph node metastasis, distant metastasis and AJCC stage was identified. FLOT2 expression may also be associated with the histological type in CRC. It was identified that FLOT2 expression was high in the majority of the mucinous adenocarcinoma of CRC (25/28). In contrast, the rate of low FLOT2 expression (58/152) did not differ from the rate of high expression (94/152) in non-mucinous adenocarcinoma. However, because only a small number of mucinous adenocarcinomas samples were obtained, it was not possible to identify a clear association between the FLOT2 expression and the histological types in CRCs $(\mathrm{P}=0.189)$. The inclusion of a greater number of mucinous adenocarcinomas samples may solve the problem.

A number of previous studies have suggested that FLOT2 serves critical functions in the progression and metastasis of several human malignant tumors, including nasopharyngeal carcinoma, gastric cancer, cervical carcinoma, non-small cell lung cancer, melanoma and breast cancer (15-17,21-24). For instance, Zhao et al (24) identified that FLOT2 is an indispensable member for transforming growth factor $\beta$ signaling, which is essential for the epithelial-mesenchymal transition (EMT) process in nasopharyngeal carcinoma metastasis. In cultured AGS and SGC7901 cells, knockdown of FLOT2 expression with specific siRNA resulted in an evident inhibition of the proliferative, migratory and invasive capabilities of the cells compared with those of control cells (21). In the present study, it was identified that the expression of FLOT2 in CRC tissues was markedly increased compared with the adjacent non-cancerous counterparts. Furthermore, correlation analysis indicated that the increased FLOT2 expression was associated with certain clinicopathological properties, including the depth of invasion, lymph node metastasis, distant metastasis and AJCC stage. Collectively, these results suggest that increased FLOT2 expression may promote CRC cell proliferation and metastasis. Further studies are required to investigate whether FLOT2 indeed modulates the proliferation and migration of CRC cells.

Furthermore, the OS and DFS rates were determined in patients with CRC with a low or high level of FLOT2, and it was identified that there was an inverse association between the level of FLOT2 and prognosis of patients. Similarly, it was identified that the patients with a high level of FLOT2 had a poorer outcome compared with those with a low expression level of FLOT2 in the pT3-4 subgroup and the AJCC stage III-IV subgroup. Nevertheless, no statistical association between the high FLOT2 expression and the shorter OS time was identified in either the pT1-2 subgroup or AJCC stage I-II subgroup. We hypothesize that this is due to the good prognosis of patients with CRC at an early stage and limited number of the clinical cases. In addition, Cox proportional hazards models revealed that high FLOT2 expression maintained its independent prognostic impact on OS. The results of the present study indicate that FLOT2 may be a predictor of poor prognosis for CRC.

It was identified that upregulation of FLOT2 was associated with poor prognosis and decreased survival of patients with CRC. Multivariate analysis indicated that the FLOT2 protein expression level could be used as an independent prognostic predictor for patients with CRC. Thus, the FLOT2 expression level may be useful for determining the prognosis and guiding 
Table II. Cox regression analysis of prognostic factors for overall survival in patients with colorectal cancer.

\begin{tabular}{|c|c|c|c|c|c|c|}
\hline \multirow[b]{2}{*}{ Variable } & \multicolumn{3}{|c|}{ Univariate } & \multicolumn{3}{|c|}{ Multivariate } \\
\hline & HR & CI $(95 \%)$ & P-value & HR & CI $(95 \%)$ & P-value \\
\hline Sex & 1.137 & $0.713-1.936$ & 0.372 & & & \\
\hline Age & 0.765 & $0.536-1.821$ & 0.427 & & & \\
\hline Tumor size & 0.634 & $0.345-1.176$ & 0.425 & & & \\
\hline Tumor location & 0.213 & $0.819-2.778$ & 0.719 & & & \\
\hline Differentiation & 0.729 & $0.467-1.306$ & 0.116 & & & \\
\hline Depth of invasion & 3.231 & $1.312-5.832$ & 0.034 & 1.442 & $0.845-1.886$ & 0.676 \\
\hline Lymph node metastasis & 3.509 & $2.399-5.442$ & 0.004 & 2.123 & $1.371-4.734$ & 0.002 \\
\hline Distant metastasis & 5.738 & $3.548-8.536$ & 0.002 & 3.631 & $2.514-6.722$ & 0.001 \\
\hline AJCC stage & 3.448 & $2.928-6.335$ & 0.021 & 1.693 & $1.021-4.561$ & 0.027 \\
\hline FLOT2 expression & 4.485 & $2.375-7.488$ & 0.001 & 2.987 & $0.962-5.135$ & 0.013 \\
\hline
\end{tabular}

HR, hazard ratio; CI, confidence interval; AJCC, American Joint Committee on Cancer; FLOT2, flotillin 2.

A

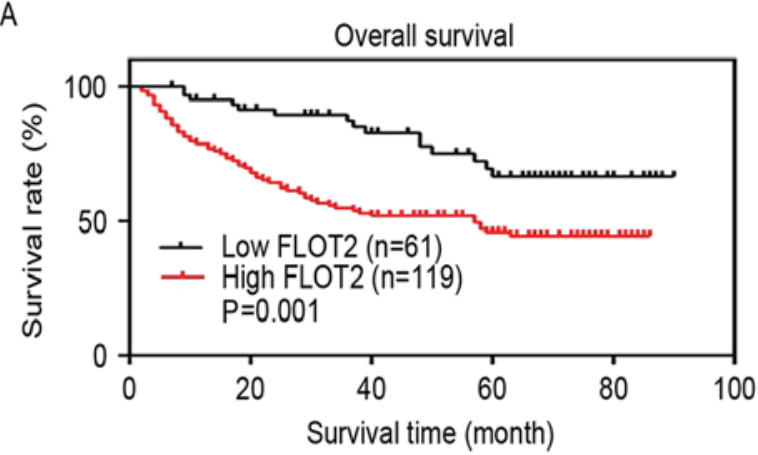

C

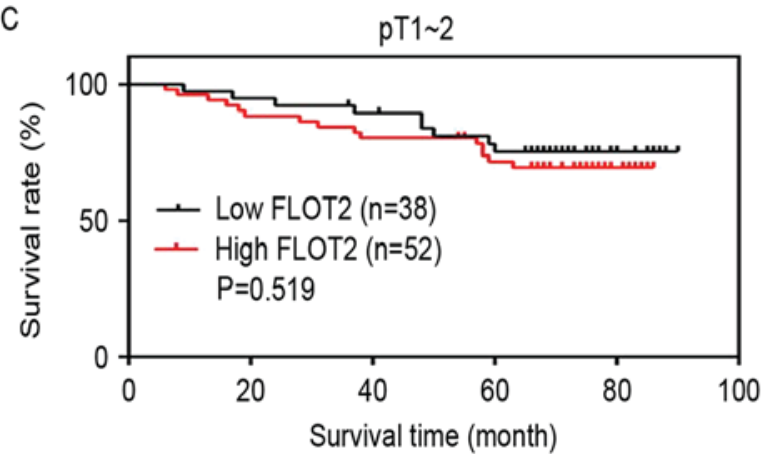

E

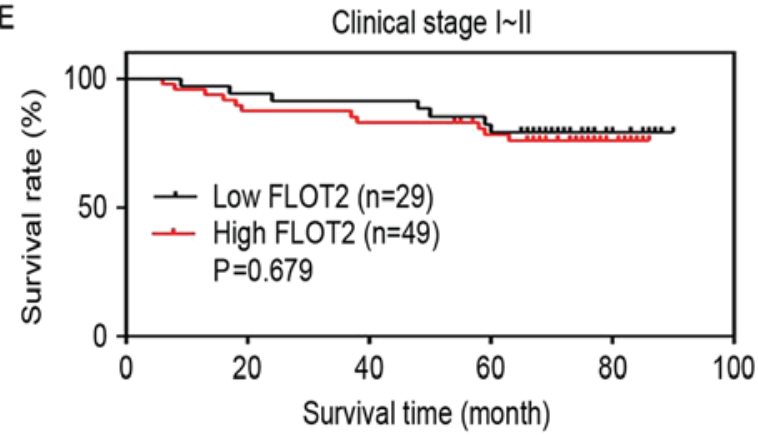

B

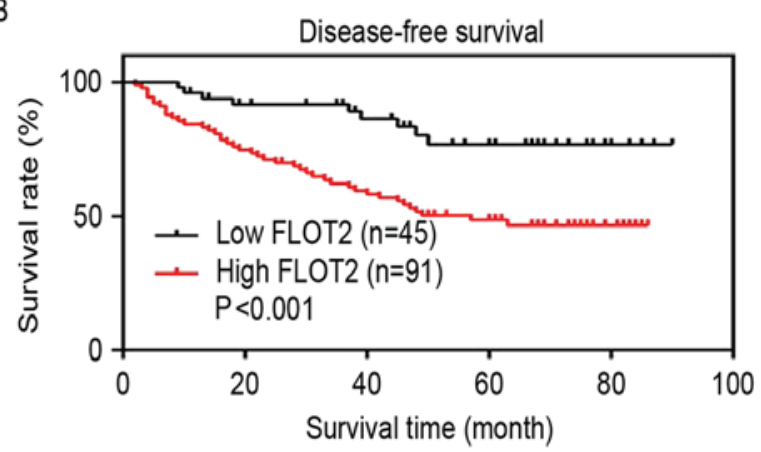

D

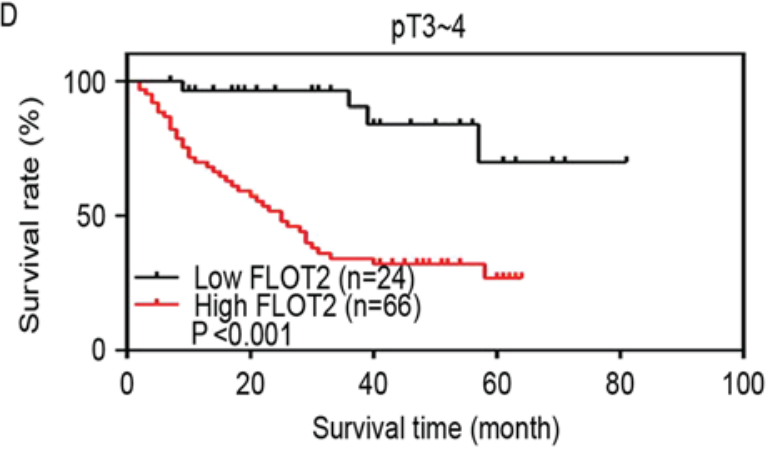

F

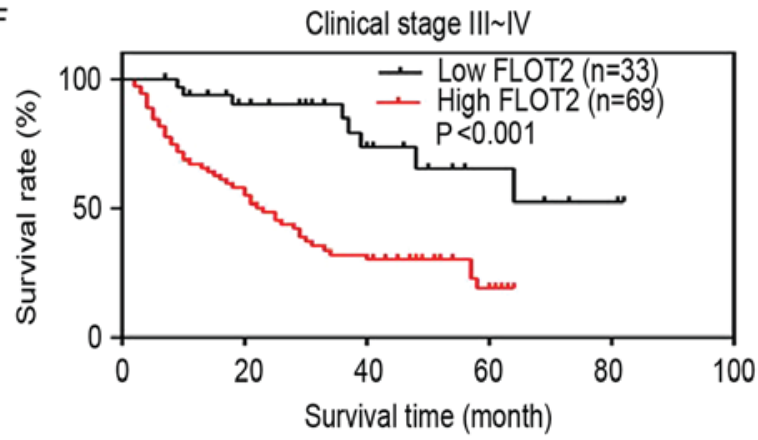

Figure 4. FLOT2 expression is association with the survival rate of patients with colorectal cancer. (A) OS and (B) 5-year DFS rates for cases with high FLOT2 expression compared with those for cases with low FLOT2 expression for all patients. (C) OS rate for cases with high FLOT2 expression compared with cases with low FLOT2 expression for patients with pT1-2 grade tumors. (D) OS rate for cases with high FLOT2 expression compared with that for cases with low FLOT2 expression for patients with pT3-4 grade tumors. (E) OS rate for cases at early clinical stages (AJCC stage I-II) with high FLOT2 expression compared with those for cases with low FLOT2 expression levels. (F) OS rate for cases at late stages (AJCC stage III-IV) with high FLOT2 expression levels compared with those for cases with low FLOT2 expression. FLOT2, flotillin-2; OS, overall survival; DFS, disease-free survival. 
the follow-up schedule in patients with CRC. FLOT2 may be useful as a biomarker for CRC diagnosis and a drug target for CRC treatment.

\section{Acknowledgements}

The authors thank Professor Sheng Liu and Professor Li Xia for providing help with pathological diagnosis and immunohistochemistry experiments.

\section{Funding}

The present study was supported by the National Natural Science Foundation of China (grant no. 201581402401).

\section{Availability of data and materials}

All data generated or analyzed during this study are included in this published article.

\section{Authors' contributions}

TL participated in the study design, literature research, data analysis and interpretation, and approved the final version of the manuscript. CC took part in the experiments, data acquisition, analysis and interpretation, and manuscript preparation and editing. QX participated in the experiments, data acquisition, analysis and interpretation. DL took part in the study concept and design, literature research, experimental procedures, data acquisition, analysis and interpretation, statistical analysis, and manuscript preparation, editing, review and final approval.

\section{Ethics approval and consent to participate}

The present study was approved by the Ethics Committee of the First Affiliated Hospital of Nanchang University (Nanchang, China).

\section{Patient consent for publication}

Not applicable.

\section{Competing interests}

The authors declare that they have no competing interests.

\section{References}

1. Torre LA, Bray F, Siegel RL, Ferlay J, Lortet-Tieulent J and Jemal A: Global cancer statistics, 2012. CA Cancer J Clin 65: 87-108, 2015.

2. Cunningham D, Atkin W, Lenz HJ, Lynch HT, Minsky B, Nordlinger B and Starling N: Colorectal cancer. Lancet 375: 1030-1047, 2010.

3. Jary M, Lecomte T, Bouché O, Kim S, Dobi E, Queiroz L, Ghiringhelli F, Etienne H, Leger J, Godet Y, et al: Prognostic value of baseline seric Syndecan-1 in initially unresectable metastatic colorectal cancer patients: A simple biological score. Int J Cancer 139: 2325-2335, 2016.

4. Barkhatov L, Fretland AA, Kazaryan AM, Rosok BI, Brudvik KW, Waage A, Bjornbeth BA, Sahakyan MA and Edwin B: Validation of clinical risk scores for laparoscopic liver resections of colorectal liver metastases: A 10-year observed follow-up study. J Surg Oncol 114: 757-763, 2016.
5. Pritchard CC and Grady WM: Colorectal cancer molecular biology moves into clinical practice. Gut 60: 116-129, 2011.

6. Simons K and Toomre D: Lipid rafts and signal transduction. Nat Rev Mol Cell Biol 1: 31-39, 2000.

7. Babuke T and Tikkanen R: Dissecting the molecular function of reggie/flotillin proteins. Eur J Cell Biol 86: 525-532, 2007.

8. Bickel PE, Scherer PE, Schnitzer JE, Oh P, Lisanti MP and Lodish HF: Flotillin and epidermal surface antigen define a new family of caveolae-associated integral membrane proteins. J Biol Chem 272: 13793-13802, 1997.

9. Banning A, Tomasovic A and Tikkanen R: Functional aspects of membrane association of reggie/flotillin proteins. Curr Protein Pept Sci 12: 725-735, 2011.

10. Lang DM, Lommel S, Jung M, Ankerhold R, Petrausch B, Laessing U, Wiechers MF, Plattner $\mathrm{H}$ and Stuermer CA: Identification of reggie-1 and reggie-2 as plasmamembrane-associated proteins which cocluster with activated GPI-anchored cell adhesion molecules in non-caveolar micropatches in neurons. J Neurobiol 37: 502-523, 1998.

11. Langhorst MF, Reuter A and Stuermer CA: Scaffolding microdomains and beyond: The function of reggie/flotillin proteins. Cell Mol Life Sci 62: 2228-2240, 2005.

12. Schulte T, Paschke KA, Laessing U, Lottspeich F and Stuermer CA: Reggie-1 and reggie-2, two cell surface proteins expressed by retinal ganglion cells during axon regeneration. Development 124: 577-587, 1997

13. Stuermer CA, Lang DM, Kirsch F, Wiechers M, Deininger SO and Plattner H: Glycosylphosphatidyl inositol-anchored proteins and fyn kinase assemble in noncaveolar plasma membrane microdomains defined by reggie-1 and -2. Mol Biol Cell 12: 3031-3045, 2001.

14. Perou CM, Sørlie T, Eisen MB, van de Rijn M, Jeffrey SS, Rees CA, Pollack JR, Ross DT, Johnsen H, Akslen LA, et al: Molecular portraits of human breast tumours. Nature 406: 747-752, 2000.

15. Hazarika P, McCarty MF, Prieto VG, George S, Babu D, Koul D, Bar-Eli M and Duvic M: Up-regulation of Flotillin-2 is associated with melanoma progression and modulates expression of the thrombin receptor protease activated receptor 1 . Cancer Res 64: 7361-7369, 2004

16. Berger T, Ueda T, Arpaia E, Chio II, Shirdel EA, Jurisica I, Hamada K, You-Ten A, Haight J, Wakeham A, et al: Flotillin-2 deficiency leads to reduced lung metastases in a mouse breast cancer model. Oncogene 32: 4989-4994, 2013.

17. Wang X, Yang Q, Guo L, Li XH, Zhao XH, Song LB and Lin HX: Flotillin-2 is associated with breast cancer progression and poor survival outcomes. J Transl Med 11: 190, 2013.

18. Lee KW, Lee SS, Kim SB, Sohn BH, Lee HS, Jang HJ, Park YY, Kopetz S, Kim SS, Oh SC and Lee JS: Significant association of oncogene YAP1 with poor prognosis and cetuximab resistance in colorectal cancer patients. Clin Cancer Res 21: 357-364, 2015.

19. Livak KJ and Schmittgen TD: Analysis of relative gene expression data using real-time quantitative PCR and the 2(-Delta Delta C(T)) method. Methods 25: 402-408, 2001.

20. Sun L, Hu S, Yu L, Guo C, Sun L, Yang Z, Qi J and Ran Y: Serum haptoglobin as a novel molecular biomarker predicting colorectal cancer hepatic metastasis. Int J Cancer 138: 2724-2731, 2016.

21. Cao K, Xie D, Cao P, Zou Q, Lu C, Xiao S, Zhou J and Peng X: SiRNA-mediated flotillin-2 (Flot2) downregulation inhibits cell proliferation, migration, and invasion in gastric carcinoma cells. Oncol Res 21: 271-279, 2014.

22. Liu Y, Lin L, Huang Z, Ji B, Mei S, Lin Y and Shen Z: High expression of flotillin-2 is associated with poor clinical survival in cervical carcinoma. Int J Clin Exp Pathol 8: 622-628, 2015.

23. Wang YL, Yao WJ, Guo L, Xi HF, Li SY and Wang ZM: Expression of flotillin-2 in human non-small cell lung cancer and its correlation with tumor progression and patient survival. Int J Clin Exp Pathol 8: 601-607, 2015.

24. Zhao L, Lin L, Pan C, Shi M, Liao Y, Bin J and Liao W: Flotillin-2 promotes nasopharyngeal carcinoma metastasis and is necessary for the epithelial-mesenchymal transition induced by transforming growth factor- $\beta$. Oncotarget 6: 9781-9793, 2015.

This work is licensed under a Creative Commons Attribution-NonCommercial-NoDerivatives 4.0 International (CC BY-NC-ND 4.0) License. 